\title{
Buyer's Equilibrium with Capacity Constraints and Restricted Mobility: a Recursive Approach
}

\author{
Gabriele Camera $^{\dagger} \quad$ Jaehong $\mathrm{Kim}^{\ddagger}$
}

November 2, 2012

\begin{abstract}
We study a decentralized trading model as in Peters (1984), where heterogeneous market participants face a trade-off between price and trade probability. We present a novel proof of existence of a unique demand vector in Nash equilibrium, based on a recursive approach that exploits the monotonicity of matching functions.
\end{abstract}

Keywords: Nash equilibrium, recursive methods, existence, heterogeneity, matching

JEL: C70, D390, D490, E390

\footnotetext{
${ }^{\dagger}$ Corresponding author. University of Basel and Chapman University. Address: Faculty of Business and Economics, Department of Macroeconomics, University of Basel, Peter Merian-Weg 6, CH - 4002 Basel, Switzerland; e-mail: gabriele.camera@unibas.ch; Tel. Tel: +41(0)612672458.

${ }^{\ddagger}$ Purdue University and University of Basel. Address: Dept. of Economics, 425 West State st., West Lafayette, IN 47907-2056, USA; e-mail: kim101@purdue.edu.
} 


\section{Introduction}

We study existence of equilibrium in the static market considered in [5], where an endogenous matching process partitions a countable population of heterogeneous sellers and homogeneous buyers in separate trading groups of different sizes. We offer a new proof of existence and uniqueness of an equilibrium demand vector.

The model is a sequential game of complete information. Each seller has a fixed amount of a good that buyers desire in fixed amounts. Buyers can compensate sellers by direct utility transfers. The interaction evolves across three stages. First, sellers simultaneously and independently advertise a price or, equivalently, a utility level promised to any buyer who trades with the seller. In the second stage, buyers see all promised utilities and then, simultaneously and independently, decide to visit one seller; in this sense, the matching process is endogenous. Frictions arise in the third stage, when matches are realized and sellers may trade only if they have been visited by at least one buyer. Because sellers may be visited by more than one buyer but are capacity constrained, they ration buyers at random. Because buyers visit exactly one seller and cannot coordinate their visits, some sellers may remain unmatched. Hence, market participants face a trade-off between price and probability of trade. ${ }^{1}$

Existence of equilibrium is usually studied in two steps. First, the equilibrium demand vector is determined in the "buyers' game," i.e., the continuation game where buyers choose which seller to visit, given a vector of promised utilities. Second, the equilibrium distribution of promised utilities is determined by studying the "sellers' game," i.e., the initial stage where sellers announce

\footnotetext{
${ }^{1}$ Models of this type have been used to study issues in IO, eg., [6, 8], and form the basis of the so-called "directed search" literature, which mostly studies labor issues, e.g., [1, 2, 3, 4].
} 
utilities taking as given the equilibrium demand vector.

This paper is about existence of equilibrium in the buyers' game: it develops a new proof of existence and uniqueness of an equilibrium demand vector. The proof is based on a recursive (or iterative) approach, and is offered as an alternative to the one developed in [5], which is based on a fixed-point argument. The proof provides an algorithm helpful to compute the equilibrium. Such methodological contribution deepens our understanding of equilibrium and present a novel avenue of analysis to study heterogeneous markets with endogenous matching.

\section{The model}

Consider an economy with finitely many players. Following the notation in [5], let $\mathcal{J}=\{1, \ldots, J\}$, with $J \geq 2$ be the set of heterogeneous sellers where heterogeneity can be multidimensional (in which case we would need an index to summarize it). Let $\mathcal{I}=\{1, \ldots, I\}$ with $I \geq 2$ be the set of homogeneous buyers.

Each seller has one indivisible object, possibly differentiated, from which she derives no utility. Each buyer desires to consume one object; let $r_{j}>$ 0 denote buyers' reservation value from consuming object $j$. Buyers, who have linear preferences ${ }^{2}$ and sellers, who may be risk-averse, play a sequential game of complete information, over three stages. In the first stage, sellers simultaneously and independently announce a price for the object they have; full commitment to the announced price is assumed. Let $\mathbf{v}=\left(v_{1}, \ldots, v_{J}\right) \subset \mathbb{R}_{+}^{J}$ denote a "promised utility," i.e., $v_{j}$ is the indirect utility for a buyer who

\footnotetext{
${ }^{2}$ The work in [7] considers risk-averse buyers.
} 
purchases the good offered by seller $j$ at the price posted by that seller. Hence, $\mathbf{v}$ is the strategy profile of all sellers. We think of $v_{j}$ as a decreasing function of the price posted by seller $j$, hence we work with $v_{j}$ instead of the price. We say that seller $j$ "posts" $v_{j} \in\left[\underline{v}_{j}, \bar{v}_{j}\right] \subset \mathbb{R}_{+}$.

In the second stage, buyers observe $\mathbf{v}$ and then simultaneously and independently choose to visit a single seller. Let $\boldsymbol{\pi}(\mathbf{v})=\left(\pi_{1}(\mathbf{v}), \ldots, \pi_{J}(\mathbf{v})\right) \in \triangle^{J-1}$ denote the strategy profile of buyers when they act symmetrically, i.e., $\pi_{j}(\mathbf{v})$ denotes the probability that any buyer chooses to visit seller $j=1, \ldots, J$ after observing $\mathbf{v}$. We call a match an encounter between one seller and $i=1, \ldots, I$ buyers.

In the third stage, matches are realized and a trade process takes place in each match. Consider a match between a generic seller and $i$ buyers; due to capacity constraints, at most one buyer trade with the seller. Let $\rho(i) \leq 1$ denote the probability that a generic buyer in the match trades with the seller. It is assumed that the assignment rule $\rho$ is match- and buyer-independent. Conditional on being visited by $i \geq 1$ buyers, the seller trades with probability $i \rho(i) \leq 1$. That is, the seller trades with at most one buyer (due to capacity constraints) and may not trade at all. ${ }^{3}$

A payoff of zero is given to players who are unmatched or do not trade. If trade takes place, then the buyer receives the promised utility $v_{j}$ and transfers utility $r_{j}-v_{j}$ to seller $j$. Thus, we can think of $r_{j}-v_{j}$ as a price. Players max-

\footnotetext{
${ }^{3}$ The work in [5] considers random rationing $\rho(i)=\frac{1}{i}$, so a buyer with no competitors trades with certainty. One can also think about cases in which buyers who are offered the good do not purchase it with some exogenous probability $\theta \in[0,1)$. This can occur for instance if buyers' valuations $r_{j}$ for the good sold by seller $j$ are i.i.d. and are realized after visiting the seller. Here, trade may fail to take place and we have $\rho(i)=\frac{1-\theta^{i}}{i}$, for all $i=1, \ldots, I$. Or there may be a seller-specific shock preventing the execution of a trade; if this occurs with probability $a_{j}$ at seller $j$, then the analysis that follows is unchanged and we simply redefine the promised utility as $\nu_{j}=a_{j} v_{j}$.
} 
imize their unconditional payoff (or expected utility) without the possibility to communicate with each other and to coordinate their actions. ${ }^{4}$

\section{The main result}

We study strongly symmetric equilibrium, i.e., an outcome in which all players of the same type adopt identical strategies in and out of equilibrium. This is the focus in the literature; see [5]. Given symmetry, we study the behavior of a generic buyer.

\subsection{Preliminaries}

Consider any seller. Let $q^{i}(I, \pi)$ denote the probability that $0 \leq i \leq I$ buyers visit this seller, when buyers identically choose to visit the seller with probability $\pi$. As a result of independent, simultaneous and symmetric choice of buyers, the distribution of buyers at any seller is binomial with parameters with $\pi$ and $I$; see [5]. Hence, $q^{i}(I, \pi)$ is a smooth of function of $\pi$, and it satisfies

$$
q^{i}(I, \pi):=\frac{I !}{i !(I-i) !} \pi^{i}(1-\pi)^{I-i}, \quad \text { for all } i=0, \ldots, I
$$

Let $\mathcal{M}(\pi)$ denote the unconditional probability that a seller trades, given $\pi$. Let $\mathcal{H}(\pi)$ denote the conditional probability that a buyer trades conditional on visiting this seller, when every other buyer visits that same seller with probability $\pi$. Hence, $\mathcal{H}\left(\pi_{j}\right) v_{j}$ is the buyer's payoff conditional on visiting

\footnotetext{
${ }^{4}$ The seller's payoff function is not needed for the results, hence is not specified. Clearly, seller's ex-post profit should decrease in the utility promised to the buyer; see [5].
} 
seller $j$. We have

$$
\begin{aligned}
\mathcal{H}(\pi) & :=\sum_{i=0}^{I-1} q^{i}(I-1, \pi) \rho(i+1) \\
\mathcal{M}(\pi) & :=\sum_{i=1}^{I} q^{i}(I, \pi) i \rho(i) .
\end{aligned}
$$

The literature typically assumes that for each $\pi$, (i) $\mathcal{M}$ is $C^{2}, \mathcal{M}^{\prime}>0$ and $\mathcal{M}^{\prime \prime}<0$; (ii) $\mathcal{H}$ is $C^{2}, \mathcal{H}^{\prime}<0$ and $\mathcal{H}^{\prime \prime}>0$; (iii) $\mathcal{H}(\pi)^{-1}$ is convex; (iv) $\mathcal{H}(\pi) v$ is quasiconcave. To prove our main result we only need monotonicity of $\mathcal{H}$; one can show that the above assumption emerge endogenously in a symmetric outcome. $^{5}$

\subsection{Recursive equilibrium in the buyers' game}

Consider the subgame where buyers choose sellers based on the posted promised utility vector $\mathbf{v}$, i.e., the "buyers' game." In symmetric equilibrium all buyers visit seller $j$ with identical probability $\pi_{j}(\mathbf{v})$ (other, non-symmetric equilibria are possible; see [2]).

Definition 1. Given $\mathbf{v}$, a symmetric equilibrium in the buyers' game is a vector $\boldsymbol{\pi}(\mathbf{v})$ such that: $\sum_{j \in \mathcal{J}} \pi_{j}(\mathbf{v})=1$; if $\pi_{j}(\mathbf{v})>0$ for $j \in \mathcal{J}$, then $\mathcal{H}\left(\pi_{j}(\mathbf{v})\right) v_{j}=$ $\max _{k \in \mathcal{J}} \mathcal{H}\left(\pi_{k}(\mathbf{v})\right) v_{k}$.

If $\pi_{j}(\mathbf{v})>0$, then the buyers' payoff from visiting that seller is no less than the payoff from visiting any other seller. Hence, if buyers randomize visits across sellers, then their payoffs from visiting any of these sellers are

\footnotetext{
${ }^{5}$ For completeness, we do so in an online Appendix. The properties of $\mathcal{M}$ are derived by direct differentiation, with some rearrangement. Mathematical induction is used to derive the properties of $\mathcal{H}$ and $1 / \mathcal{H}$.
} 
equivalent, i.e.,

$$
\mathcal{H}\left(\pi_{j}(\mathbf{v})\right) v_{j}=\mathcal{H}\left(\pi_{k}(\mathbf{v})\right) v_{k} \quad \text { for all } \quad j, k \in \Gamma(\mathbf{v}):=\left\{i \in \mathcal{J} \mid \pi_{i}(\mathbf{v})>0\right\}
$$

Given $\mathbf{v}$, the set $\Gamma(\mathbf{v})$ is the collection of sellers who are "in the market," i.e., the set of sellers that may be visited by a buyer. The complementary set $\mathcal{J} \backslash \Gamma(\mathbf{v})$ contains "idle" sellers, i.e., sellers that post a specific utility $v$ but are certain not to be visited by any buyer. Given Definition 1

$$
\begin{array}{ll}
\bar{H}(\mathbf{v}):=\mathcal{H}\left(\pi_{k}(\mathbf{v})\right) v_{k} & \text { for } k \in \Gamma(\mathbf{v}) \\
V\left(v_{k}\right):=\mathcal{H}(0) v_{k} \leq \bar{H}(\mathbf{v}) & \text { for } k \in \mathcal{J} \backslash \Gamma(\mathbf{v}) .
\end{array}
$$

$\bar{H}(\mathbf{v})$ is the expected utility derived from visiting any sellers who is in the market, in buyers' equilibrium; this payoff is independent of $k$ for $k \in \Gamma(\mathbf{v})$. Instead, $V\left(v_{k}\right)$ denotes the expected utility derived from visiting idle seller $k$; this out-of-equilibrium payoff depends on $k$.

Proposition 1. Fix $\mathbf{v} \neq 0$. There exists a unique equilibrium vector $\boldsymbol{\pi}(\mathbf{v})$ for the buyers' game.

Proof. Fix $\mathbf{v} \neq 0$, and consider the choice $\boldsymbol{\pi}(\mathbf{v})$ of a generic buyer. The proof is in two parts. First, construct a demand distribution $\boldsymbol{\pi}$ through an iterative process. Second, prove that this equilibrium $\boldsymbol{\pi}$ is unique by means of contradiction.

Without loss of generality, let $v_{1} \geq v_{2} \geq \ldots \geq v_{J}$ with $v_{1}>0$. Suppose the buyer considers sellers one at a time, according to an iterative process. Let $\eta_{j}^{s}(\mathbf{v})$ denote the probability that this generic buyer visits seller $j$ when the first $s=1, \ldots, J$ sellers have been considered for a possible visit. Hence, 
$\sum_{j=1}^{s} \eta_{j}^{s}(\mathbf{v})=1$, so that $\eta_{1}^{1}(\mathbf{v})=1$. Since promised utilities $\mathbf{v}$ are fixed, we omit $\mathbf{v}$ as an argument when understood.

For the initial step of the iterative decision process, define $\eta^{1}$ as the $J$-dimensional vector

$$
\boldsymbol{\eta}^{1}:=\left(\eta_{1}^{1}, 0, \ldots, 0\right)
$$

This means that when only seller one is being considered for a visit, then the buyer goes there with probability 1 because $v_{1}>0$. Since the sequence $v_{j}$ is decreasing, for $2 \leq s \leq J$ recursively define

$\boldsymbol{\eta}^{s}\left(\boldsymbol{\eta}^{s-1}\right)= \begin{cases}\left(\eta_{1}^{s}, \ldots, \eta_{s}^{s}, 0, \ldots, 0\right) \text { s.t. } \mathcal{H}\left(\eta_{j}^{s}\right) v_{j}=\mathcal{H}\left(\eta_{s}^{s}\right) v_{s}, j \leq s, & \text { if } V\left(v_{s}\right)>\mathcal{H}\left(\eta_{1}^{s-1}\right) v_{1} \\ \left(\eta_{1}^{s-1}, \ldots, \eta_{s-1}^{s-1}, 0, \ldots, 0\right), & \text { otherwise }\end{cases}$

Start by noting that $\boldsymbol{\eta}=\boldsymbol{\eta}^{J}$ satisfies Definition 1, therefore $\boldsymbol{\eta}$ is a candidate equilibrium vector of buyers' choices. It should be clear that continuity of $\mathcal{H}$, $\mathcal{H}^{\prime}<0$ and the recursive construction of $\boldsymbol{\eta}$ ensure that $\boldsymbol{\eta}$ exists. We also have that

$$
\mathcal{H}\left(\eta_{j}^{s-1}\right) v_{j} \leq \mathcal{H}\left(\eta_{j}^{s}\right) v_{j} \text { for } j=1, \ldots, s-1
$$

This means that the vector $\boldsymbol{\eta}$ maximizes the payoff of the generic buyer.

We claim that if any other vector of buyers' choices $\boldsymbol{\pi}$ exists that satisfies Definition 1, then $\boldsymbol{\pi}=\boldsymbol{\eta}$. That is, a unique equilibrium demand distribution exists. To prove it, suppose by means of contradiction that $\boldsymbol{\pi} \neq \boldsymbol{\eta}$. Let $\Gamma_{\boldsymbol{\pi}}(\mathbf{v})$ and $\Gamma_{\boldsymbol{\eta}}(\mathbf{v})$ denote the sets of sellers who are in the market associated to $\boldsymbol{\pi}$ and $\boldsymbol{\eta}$. There are three cases to consider.

Case 1: $\Gamma_{\pi}=\Gamma_{\eta}=\Gamma$

If $\boldsymbol{\pi} \neq \boldsymbol{\eta}$ then $\pi_{i} \neq \eta_{i}$ for some $i \in \Gamma$. Without loss of generality, consider 
$\pi_{i}<\eta_{i}$. If $\mathcal{H}\left(\pi_{i}\right) v_{i}>\mathcal{H}\left(\eta_{i}\right) v_{i}$, then

$$
\mathcal{H}\left(\pi_{j}\right) v_{j}>\mathcal{H}\left(\eta_{j}\right) v_{j} \quad \text { for all } j \in \Gamma=\Gamma_{\boldsymbol{\pi}}=\Gamma_{\boldsymbol{\eta}}
$$

because $\boldsymbol{\pi}$ and $\boldsymbol{\eta}$ are assumed to satisfy Definition 1 and, in particular the indifference condition $\mathcal{H}\left(\pi_{i}\right) v_{i}=\max _{j \in \mathcal{J}} \mathcal{H}\left(\pi_{j}\right) v_{j}$.

Now note that $\mathcal{H}^{\prime}<0$ implies $\pi_{j}<\eta_{j}$ for all $j \in \Gamma$. Hence, $\sum_{i \in \Gamma_{\boldsymbol{\pi}}} \pi_{i}=1<\sum_{i \in \Gamma_{\boldsymbol{\eta}}} \eta_{i}$. This gives us the desired contradiction. Hence $\boldsymbol{\pi}=\boldsymbol{\eta}$.

Case 2: $\Gamma_{\pi} \subsetneq \Gamma_{\eta}$

We consider only this inclusion because the other way around simply means changing the role of $\eta$ and $\pi$. This case is studied in two steps. First we claim that $\eta_{i}<\pi_{i}$ for all $i \in \Gamma_{\boldsymbol{\pi}}$; intuitively, if there are less idle sellers under $\boldsymbol{\eta}$ than $\boldsymbol{\pi}$, then the probability of visiting any seller cannot be greater under $\boldsymbol{\eta}$ than $\boldsymbol{\pi}$. Suppose by means of contradiction that $\eta_{i} \geq \pi_{i}$ for some $i \in \Gamma_{\pi}$. Then, $\eta_{k} \geq \pi_{k}$ for all $k \in \Gamma_{\boldsymbol{\pi}}$ by the same argument developed in case 1 above (indifference). This gives us a contradiction because $\sum_{i \in \Gamma_{\eta}} \eta_{i}>1=\sum_{i \in \Gamma_{\pi}} \pi_{i}$. Hence $\eta_{i}<\pi_{i}$ for all $i \in \Gamma_{\boldsymbol{\pi}}$.

Now we show that $\boldsymbol{\pi} \neq \boldsymbol{\eta}$ is impossible. Fix $i, j \in \Gamma_{\boldsymbol{\eta}}, i \in \Gamma_{\boldsymbol{\pi}}, j \in \mathcal{J} \backslash \Gamma_{\boldsymbol{\pi}}$. Notice that the last two relations imply $\mathcal{H}\left(\pi_{i}\right) v_{i} \geq \mathcal{H}(0) v_{j}$, hence $v_{i}>v_{j}$. Now recall that $\eta_{i}<\pi_{i}$ and $\mathcal{H}^{\prime}<0$. Therefore we have the contradiction

$$
\overbrace{\mathcal{H}(0) v_{j}>\mathcal{H}\left(\eta_{i}\right) v_{i}}^{\text {seller } j \text { is in the market under } \boldsymbol{\eta}} \geq \overbrace{\mathcal{H}\left(\pi_{i}\right) v_{i} \geq \mathcal{H}(0) v_{j}}^{\text {seller } j \text { is idle under } \boldsymbol{\pi}} .
$$

Therefore $\boldsymbol{\pi}=\boldsymbol{\eta}$.

Case 3: Neither $\Gamma_{\boldsymbol{\pi}} \subset \Gamma_{\boldsymbol{\eta}}$ nor $\Gamma_{\boldsymbol{\pi}} \supset \Gamma_{\boldsymbol{\eta}}$ Consider sellers $i$ and $j$ such that $i \in \Gamma_{\boldsymbol{\eta}} \backslash \Gamma_{\boldsymbol{\pi}}$ and some $j \in \Gamma_{\boldsymbol{\pi}} \backslash \Gamma_{\boldsymbol{\eta}}$. Without 
loss of generality choose $i$ and $j$ so that $v_{j} \leq v_{i}$. Since $j \in \Gamma_{\boldsymbol{\pi}}$ and $i \in \mathcal{J} \backslash \Gamma_{\boldsymbol{\pi}}$, we have

$$
\overbrace{\mathcal{H}\left(\pi_{j}\right) v_{j} \geq \mathcal{H}(0) v_{i}}^{\text {seller } i \text { is idle under } \boldsymbol{\pi}} \text {, i.e. } v_{j}>v_{i} \text {. }
$$

This gives us a desired contradiction which completes the proof that $\boldsymbol{\pi}=\boldsymbol{\eta}$.

\section{References}

[1] Albrecht, J., Gautier, P. A. and Vroman S. (2006). Equilibrium directed search with multiple applications. Review of Economic Studies, 73 (4), 869-891.

[2] Burdett, K., Shi, S. and Wright, R. (2001). Pricing and matching with frictions. Journal of Political Economy, 109 (5), 1060-1085.

[3] Camera, G. and C. Selcuk (2008). Price dispersion with directed search. Journal of the European Economic Association, 7 (6), 1193-1224.

[4] Montgomery, J. (1991). Equilibrium wage dispersion and inter-industry wage differentials. Quarterly Journal of Economic, 163180.

[5] Peters, M. (1984). Bertrand equilibrium with capacity constraints and restricted mobility. Econometrica, 52 (5), 1117-1127.

[6] Peters, M. (1984). Restrictions on Price Advertising. Journal of Political Economy, 92 (3), 472-485.

[7] Selcuk, C (2012). Trading Mechanism Selection with Directed Search when Buyers are Risk Averse. Economics Letters 115 (2), 207-210.

[8] Virag, G (2011). High profit equilibria in directed search models. Games and Economic Behavior, 71, 224-234. 


\section{Supplementary Material: Endogenizing the regularity properties}

Key properties of matching and payoff functions are usually assumed in the literature to prove existence of equilibrium in the entire game. ${ }^{1}$

Here, we show that such properties emerge endogenously in a symmetric outcome. In doing so, we consider $\rho(i)=\frac{1-\theta^{i}}{i}$, for all $i=1, \ldots, I$, as it generalizes the typical case where $\theta=0$.

Proposition. Let $q^{i}(I, \pi):=\frac{I !}{i !(I-i) !} \pi^{i}(1-\pi)^{I-i}$ for all $i=0, \ldots, I$ and $\rho(i)=\frac{1-\theta^{i}}{i}$, for all $i=1, \ldots, I$. For each $\pi \in[0,1]$, we have

- $\mathcal{M}(\pi)$ is twice continuously differentiable, strictly increasing, and concave.

- $\mathcal{H}(\pi)$ is twice continuously differentiable, strictly decreasing, and convex.

- $\mathcal{H}(\pi)^{-1}$ is convex, i.e. $2\left(\mathcal{H}^{\prime}(\pi)\right)^{2}-\mathcal{H}^{\prime \prime}(\pi) \mathcal{H}(\pi) \geq 0$

- $\mathcal{H}(\pi) v$ is quasiconcave.

Proof. Consider a generic seller.

The function $\mathcal{M}(\pi)$. Notice that

$$
\begin{aligned}
\frac{I \pi}{i+1} q^{i}(I-1, \pi) & =\frac{I \pi}{i+1} \cdot \frac{(I-1) !}{i !(I-1-i) !} \pi^{i}(1-\pi)^{I-1-i} \\
& =\frac{I !}{(i+1) !(I-(i+1)) !} \pi^{i+1}(1-\pi)^{I-(i+1)}=q^{i+1}(I, \pi) .
\end{aligned}
$$

From (2) in the paper, the functions $\mathcal{M}(\pi)$ and $\mathcal{H}(\pi)$ are twice continuously differentiable, because $q^{i}(I, \pi)$ is smooth in $p$ for all $i$. Now notice

$$
\begin{aligned}
\mathcal{M}(\pi) & =\sum_{i=1}^{I} q^{i}(I, \pi)-\sum_{i=1}^{I} q^{i}(I, \pi) \theta^{i}=1-q^{0}(I, \pi)-\sum_{i=1}^{I} q^{i}(I, \pi) \theta^{i} \\
& =1-\sum_{i=0}^{I} q^{i}(I, \pi) \theta^{i}=1-\sum_{i=0}^{I}\left(\begin{array}{c}
I \\
i
\end{array}\right)(\pi \theta)^{i}(1-\pi)^{I-i} \\
& =1-(1-(1-\theta) \pi)^{I}
\end{aligned}
$$

\footnotetext{
${ }^{1}$ For a recent example consider properties (i)-(iii) in [1, Assumption 2].
} 
where the last line follows from the binomial formula. It is clear that $\mathcal{M}^{\prime}(\pi)>0$ and $\mathcal{M}^{\prime \prime}(\pi)<0$ by direct differentiation.

The functions $\mathcal{H}(\pi)$ and $1 / \mathcal{H}(\pi)$. Without loss of generality, set $\theta=0$. Thus

$$
\begin{aligned}
\mathcal{H}(\pi) & =\frac{\mathcal{M}(\pi)}{I \pi}=\frac{1-(1-\pi)^{I}}{I \pi} \\
\mathcal{H}^{\prime}(\pi) & =\frac{1}{I \pi^{2}}\left[(1-\pi)^{I-1}(1-\pi+I \pi)-1\right] .
\end{aligned}
$$

- We show that $\mathcal{H}^{\prime}(\pi)<0$ for $I \geq 2$.

The proof is by induction on $I$. Define the term

$$
A(\pi, I):=(1-\pi)^{I-1}(1-\pi+I \pi)-1 .
$$

We have

$$
\mathcal{H}^{\prime}(\pi)=\frac{1}{I \pi^{2}} A(\pi, I)
$$

Clearly $A(\pi, 1) \leq 0$. Consider the following induction hypothesis: $A(\pi, I)<0$ for some $I \geq 2$. We need to prove that $A(\pi, I+1)<0$. We have

$$
\begin{aligned}
A(\pi, I+1) & =(1-\pi)^{I}(1-\pi+I \pi+\pi)-1=(1-\pi)^{I-1}(1-\pi)(1+I \pi)-1 \\
& =(1-\pi)^{I-1}\left(1-\pi+I \pi-I \pi^{2}\right)-1<(1-\pi)^{I-1}(1-\pi+I \pi)-1 \\
& =A(\pi, I)
\end{aligned}
$$

Hence $A(\pi, I)<0$ for all $I \geq 1$. It follows that $\mathcal{H}^{\prime}(\pi)<0$.

- We show that $\mathcal{H}^{\prime \prime}(\pi)>0$ for $I \geq 2$. By direct differentiation we have:

$$
\mathcal{H}^{\prime \prime}(\pi)=\frac{\pi}{I \pi^{4}}\left\{A^{\prime}(\pi, I) \pi-2 A(\pi, I)\right\}
$$

where

$$
A^{\prime}(\pi, I):=-I(I-1)(1-\pi)^{I-2} \pi .
$$

Hence, if $A^{\prime}(\pi, I) \pi-2 A(\pi, I)>0$, then $\mathcal{H}^{\prime \prime}(\pi)>0$. The proof is by induction on $I$ :

$$
A^{\prime}(\pi, I) \pi-2 A(\pi, I)=-I(I-1)(1-\pi)^{I-2} \pi^{2}-2(1-\pi)^{I-1}(1-\pi+I \pi)+2
$$

For $I=2$ we have $A^{\prime} \pi-2 A=0$. For the induction hypothesis suppose $A^{\prime} \pi-2 A>0$ for some $I \geq 3$, that is

$$
2\left[(1-\pi)^{I-1}(1-\pi+I \pi)-1\right]<-I(I-1)(1-\pi)^{I-2} \pi^{2}
$$


We need to show that this is true for $I+1$, i.e.,

$$
\begin{aligned}
2(1-\pi)^{I}(1-\pi+I \pi+\pi)-2 & \leq-I(I+1)(1-\pi)^{I-1} \pi^{2} \\
2\left[(1-\pi)^{I-1}(1-\pi)(1+I \pi)-1\right] & \leq-I(I-1)(1-\pi)^{I-1} \pi^{2}-2 I(1-\pi)^{I-1} \pi^{2} \\
2\left[(1-\pi)^{I-1}(1-\pi+I \pi)-1\right] & \leq-I(I-1)(1-\pi)^{I-1} \pi^{2}
\end{aligned}
$$

By virtue of the induction hypothesis we have

$$
2\left[(1-\pi)^{I-1}(1-\pi+I \pi)-1\right]<-I(I-1)(1-\pi)^{I-2} \pi^{2}<-I(I-1)(1-\pi)^{I-1} \pi^{2}
$$

so the statement is true for $I+1$.

- We prove $\left.\mathcal{H}^{\prime \prime}(\pi) \mathcal{H}(\pi)-2\left(\mathcal{H}^{\prime} \pi\right)\right)^{2}<0$.

Notice that

$$
\begin{aligned}
& \left.\mathcal{H}^{\prime \prime}(\pi) \mathcal{H}(\pi)-2\left(\mathcal{H}^{\prime} \pi\right)\right)^{2} \\
& \quad=\frac{\pi}{I \pi^{4}}\left\{A^{\prime}(\pi, I) p-2 A(\pi, I)\right\} \frac{1-(1-\pi)^{I}}{I \pi}-2\left(\frac{1}{I \pi^{2}}\right)^{2}\left[(1-\pi)^{I-1}(1-\pi+I \pi)-1\right]^{2} \\
& \quad \propto\left[A^{\prime}(\pi, I) \pi-2 A(\pi, I)\right]\left[1-(1-\pi)^{I}\right]-2[A(\pi, I)]^{2}
\end{aligned}
$$

We want to prove that

$$
\left[A^{\prime}(\pi, I) \pi-2 A(\pi, I)\right]\left[1-(1-\pi)^{I}\right]-2[A(\pi, I)]^{2}<0 .
$$

Using the definition for $A^{\prime}$ and $A$ this can be rearranged as

$$
-(I-1) \pi\left[1-(1-\pi)^{I}\right]<2 A(\pi, I)(1-\pi) .
$$

Again, we use the proof by induction. Notice that for $I=2$ the inequality above holds. For the induction hypothesis, suppose it also holds for some $I \geq 2$. Then we must show that it holds for $I+1$, i.e.

$$
-I \pi\left[1-(1-\pi)^{I+1}\right]<2 A(\pi, I+1)(1-\pi) .
$$

This inequality is rearranged as

$$
\begin{aligned}
-I \pi\left[1-(1-\pi)^{I}\right]+I \pi^{2}(1-\pi)^{I} & <(1-\pi) 2\left[(1-\pi)^{I-1}(1-\pi+I \pi)-1\right] \\
& =2(1-\pi) A(\pi, I)
\end{aligned}
$$


The left hand side can be rearranged as follows:

$$
\begin{aligned}
-(I-1) \pi & {\left[1-(1-\pi)^{I}\right]-\pi\left[1-(1-\pi)^{I}\right]+I \pi^{2}(1-\pi)^{I} } \\
& =-(I-1) \pi\left[1-(1-\pi)^{I}\right]+\pi\left[-1+(1-\pi)^{I-1}(1-\pi+I \pi)-I \pi^{2}(1-\pi)^{I-1}\right] \\
& =-(I-1) \pi\left[1-(1-\pi)^{I}\right]+\pi\left[A(I, \pi)-I \pi^{2}(1-\pi)^{I-1}\right],
\end{aligned}
$$

therefore it is smaller than $2(1-\pi) A(\pi, I)$ because (i) $A(\pi, I)-I \pi^{2}(1-$ $\pi)^{I-1}<0$ due to $A(\pi, I) \leq 0$, and (ii) by induction hypothesis above. Hence $\mathcal{H}^{\prime \prime}(\pi) \mathcal{H}(\pi)-2\left(\mathcal{H}^{\prime}(\pi)\right)^{2}<0$ for all $I \geq 2$.

Quasiconcavity of $\mathcal{H}(\pi) v$. If $\mathcal{H}(\pi) v=r \geq 0$, then the superior set is defined by $S_{r}=\{(\pi, v) \in[0,1] \times[\underline{v}, \bar{v}]: \mathcal{H}(\pi) v \geq r\}$. The set $S_{r}$ is convex because if $(\pi, v),\left(\pi^{\prime}, v^{\prime}\right) \in S_{r}$ then for $\lambda \in(0,1)$, we have

$$
\frac{r}{\mathcal{H}\left(\pi^{\lambda}\right)} \leq \lambda \frac{r}{\mathcal{H}\left(\pi^{\prime}\right)}+(1-\lambda) \frac{r}{\mathcal{H}(\pi)} \leq \lambda v^{\prime}+(1-\lambda) v
$$

where $\pi^{\lambda}=\lambda \pi^{\prime}+(1-\lambda) \pi$. The first line follows from convexity of $\frac{1}{\mathcal{H}(\pi)}$; the second line follows from $(\pi, v),\left(\pi^{\prime}, v^{\prime}\right) \in S_{r}$.

\section{References}

[1] Galenianos, M. and P. Kircher, (2012). On the Game-theoretic Foundations of Competitive Search Equilibrium. International Economic Review, 53 (1), 121. 
2012

\section{Economic Science Institute Working Papers}

12-27 Camera, G., Casari, M., and Bigoni, M. Binding Promises and Cooperation Among Strangers.

12-26 Schniter, E., Shields, T. and Dickhaut, J. Ageism \& Cooperation.

12-25 Gjerstad, S. and Smith, V. Balance Sheet Crises: Causes, Consequences and Responses.

12-24 Gómez-Miñambres, J., Corgnet, B. and Hernán-Gonzalez, R. Goal Setting and Monetary Incentives: When Large Stakes Are Not Enough.

12-23 Clots-Figueras, I., Hernán González, R., and Kujal, P. Asymmetry and Deception in the Investment Game.

12-22 Dechenaux, E., Kovenock, D. and Sheremeta, R. A Survey of Experimental Research on Contests, All-Pay Auctions and Tournaments.

12-21 Rubin, J. and Sheremeta, R. Principal-Agent Settings with Random Shocks.

12-20 Gómez-Miñambres, J. and Schniter, E. Menu-Dependent Emotions and Self-Control.

12-19 Schniter, E., Sheremeta, R., and Sznycer, D. Building and Rebuilding Trust with Promises and Apologies.

12-18 Shields, T. and Xin, B. Higher-order Beliefs in Simple Trading Models.

12-17 Pfeiffer, G. and Shields, T. Performance-Based Compensation and Firm Value: Experimental evidence.

12-16 Kimbrough, E. and Sheremeta, R. Why Can't We Be Friends? Entitlements, bargaining, and conflict.

12-15 Mago, S., Savikhin, A., and Sheremeta, R. Facing Your Opponents: Social identification and information feedback in contests.

12-14 McCarter, M., Kopelman, S., Turk, T. and Ybarra, C. Too Many Cooks Spoil the Broth: How the tragedy of the anticommons emerges in organizations.

12-13 Chowdhury, S., Sheremeta, R. and Turocy, T. Overdissipation and Convergence in Rent-seeking Experiments: Cost structure and prize allocation rules.

12-12 Bodsky, R., Donato, D., James, K. and Porter, D. Experimental Evidence on the Properties of the California's Cap and Trade Price Containment Reserve. 
12-11 Branas-Garza, P., Espin, A. and Exadaktylos, F. Students, Volunteers and Subjects: Experiments on social preferences.

12-10 Klose, B. and Kovenock, D. Extremism Drives Out Moderation.

12-09 Buchanan, J. and Wilson, B. An Experiment on Protecting Intellectual Property.

12-08 Buchanan, J., Gjerstad, S. and Porter, D. Information Effects in Multi-Unit Dutch Auctions.

12-07 Price, C. and Sheremeta, R. Endowment Origin, Demographic Effects and Individual Preferences in Contests.

12-06 Magoa, S. and Sheremeta, R. Multi-Battle Contests: An experimental study.

12-05 Sheremeta, R. and Shields, T. Do Liars Believe? Beliefs and Other-Regarding Preferences in Sender-Receiver Games.

12-04 Sheremeta, R., Masters, W. and Cason. T. Winner-Take-All and Proportional-Prize Contests: Theory and experimental results.

12-03 Buchanan, J., Gjerstad, S. and Smith, V. There's No Place Like Home.

12-02 Corgnet, B. and Rodriguez-Lara, I. Are you a Good Employee or Simply a Good Guy? Influence Costs and Contract Design.

12-01 Kimbrough, E. and Sheremeta, R. Side-Payments and the Costs of Conflict.

2011

11-20 Cason, T., Savikhin, A. and Sheremeta, R. Behavioral Spillovers in Coordination Games.

11-19 Munro, D. and Rassenti, S. Combinatorial Clock Auctions: Price direction and performance.

11-18 Schniter, E., Sheremeta, R., and Sznycer, D. Restoring Damaged Trust with Promises, Atonement and Apology.

11-17 Brañas-Garza, P., and Proestakis, A. Self-discrimination: A field experiment on obesity.

11-16 Brañas-Garza, P., Bucheli, M., Paz Espinosa, M., and García-Muñoz, T. Moral Cleansing and Moral Licenses: Experimental evidence.

11-15 Caginalp, G., Porter, D., and Hao, L. Asset Market Reactions to News: An experimental study.

11-14 Benito, J., Branas-Garz, P., Penelope Hernandez, P., and Sanchis Llopis, J. Strategic Behavior in Schelling Dynamics: A new result and experimental evidence.

11-13 Chui, M., Porter, D., Rassenti, S. and Smith, V. The Effect of Bidding Information in Ascending Auctions. 
11-12 Schniter, E., Sheremeta, R. and Shields, T. Conflicted Minds: Recalibrational emotions following trust-based interaction.

11-11 Pedro Rey-Biel, P., Sheremeta, R. and Uler, N. (Bad) Luck or (Lack of) Effort?: Comparing social sharing norms between US and Europe.

11-10 Deck, C., Porter, D., and Smith, V. Double Bubbles in Assets Markets with Multiple Generations.

11-09 Kimbrough, E., Sheremeta, R., and Shields, T. Resolving Conflicts by a Random Device.

11-08 Brañas-Garza, P., García-Muñoz, T., and Hernan, R. Cognitive effort in the Beauty Contest Game.

11-07 Grether, D., Porter, D., and Shum, M. Intimidation or Impatience? Jump Bidding in On-line Ascending Automobile Auctions.

11-06 Rietz, T., Schniter, E., Sheremeta, R., and Shields, T. Trust, Reciprocity and Rules.

11-05 Corgnet, B., Hernan-Gonzalez, R., and Rassenti, S. Real Effort, Real Leisure and Real-time Supervision: Incentives and peer pressure in virtual organizations.

11-04 Corgnet, B. and Hernán-González R. Don’t Ask Me If You Will Not Listen: The dilemma of participative decision making.

11-03 Rietz, T., Sheremeta, R., Shields, T., and Smith, V. Transparency, Efficiency and the Distribution of Economic Welfare in Pass-Through Investment Trust Games.

11-02 Corgnet, B., Kujal, P. and Porter, D. The Effect of Reliability, Content and Timing of Public Announcements on Asset Trading Behavior.

11-01 Corgnet, B., Kujal, P. and Porter, D. Reaction to Public Information in Markets: How much does ambiguity matter?

\section{0}

10-23 Sheremeta, R. Perfect-Substitutes, Best-Shot, and Weakest-Link Contests between Groups.

10-22 Mago, S., Sheremeta, R., and Yates, A. Best-of-Three Contests: Experimental evidence.

10-21 Kimbrough, E. and Sheremeta, R. Make Him an Offer He Can't Refuse: Avoiding conflicts through side payments.

10-20 Savikhim, A. and Sheremeta, R. Visibility of Contributions and Cost of Inflation: An experiment on public goods.

10-19 Sheremeta, R. and Shields, T. Do Investors Trust or Simply Gamble?

10-18 Deck, C. and Sheremeta, R. Fight or Flight? Defending Against Sequential Attacks in the Game of Siege. 
10-17 Deck, C., Lin, S. and Porter, D. Affecting Policy by Manipulating Prediction Markets:

Experimental evidence.

10-16 Deck, C. and Kimbrough, E. Can Markets Save Lives? An Experimental Investigation of a Market for Organ Donations.

10-15 Deck, C., Lee, J. and Reyes, J. Personality and the Consistency of Risk Taking Behavior: Experimental evidence.

10-14 Deck, C. and Nikiforakis, N. Perfect and Imperfect Real-Time Monitoring in a Minimum-Effort Game.

10-13 Deck, C. and Gu, J. Price Increasing Competition? Experimental Evidence.

10-12 Kovenock, D., Roberson, B., and Sheremeta, R. The Attack and Defense of Weakest-Link Networks.

10-11 Wilson, B., Jaworski, T., Schurter, K. and Smyth, A. An Experimental Economic History of Whalers' Rules of Capture.

10-10 DeScioli, P. and Wilson, B. Mine and Thine: The territorial foundations of human property.

10-09 Cason, T., Masters, W. and Sheremeta, R. Entry into Winner-Take-All and Proportional-Prize Contests: An experimental study.

10-08 Savikhin, A. and Sheremeta, R. Simultaneous Decision-Making in Competitive and Cooperative Environments.

10-07 Chowdhury, S. and Sheremeta, R. A generalized Tullock contest.

10-06 Chowdhury, S. and Sheremeta, R. The Equivalence of Contests.

10-05 Shields, T. Do Analysts Tell the Truth? Do Shareholders Listen? An Experimental Study of Analysts' Forecasts and Shareholder Reaction.

10-04 Lin, S. and Rassenti, S. Are Under- and Over-reaction the Same Matter? A Price Inertia based Account.

10-03 Lin, S. Gradual Information Diffusion and Asset Price Momentum.

10-02 Gjerstad, S. and Smith, V. Household Expenditure Cycles and Economic Cycles, 1920-2010.

10-01 Dickhaut, J., Lin, S., Porter, D. and Smith, V. Durability, Re-trading and Market Performance.

2009

09-11 Hazlett, T., Porter, D., and Smith, V. Radio Spectrum and the Disruptive Clarity OF Ronald Coase. 
09-10 Sheremeta, R. Expenditures and Information Disclosure in Two-Stage Political Contests.

09-09 Sheremeta, R. and Zhang, J. Can Groups Solve the Problem of Over-Bidding in Contests?

09-08 Sheremeta, R. and Zhang, J. Multi-Level Trust Game with "Insider" Communication.

09-07 Price, C. and Sheremeta, R. Endowment Effects in Contests.

09-06 Cason, T., Savikhin, A. and Sheremeta, R. Cooperation Spillovers in Coordination Games.

09-05 Sheremeta, R. Contest Design: An experimental investigation.

09-04 Sheremeta, R. Experimental Comparison of Multi-Stage and One-Stage Contests.

09-03 Smith, A., Skarbek, D., and Wilson, B. Anarchy, Groups, and Conflict: An experiment on the emergence of protective associations.

09-02 Jaworski, T. and Wilson, B. Go West Young Man: Self-selection and endogenous property rights.

09-01 Gjerstad, S. Housing Market Price Tier Movements in an Expansion and Collapse.

\section{8}

08-09 Dickhaut, J., Houser, D., Aimone, J., Tila, D. and Johnson, C. High Stakes Behavior with Low Payoffs: Inducing preferences with Holt-Laury gambles.

08-08 Stecher, J., Shields, T. and Dickhaut, J. Generating Ambiguity in the Laboratory.

08-07 Stecher, J., Lunawat, R., Pronin, K. and Dickhaut, J. Decision Making and Trade without Probabilities.

08-06 Dickhaut, J., Lungu, O., Smith, V., Xin, B. and Rustichini, A. A Neuronal Mechanism of Choice.

08-05 Anctil, R., Dickhaut, J., Johnson, K., and Kanodia, C. Does Information Transparency Decrease Coordination Failure?

08-04 Tila, D. and Porter, D. Group Prediction in Information Markets With and Without Trading Information and Price Manipulation Incentives.

08-03 Thomas, C. and Wilson, B. Horizontal Product Differentiation in Auctions and Multilateral Negotiations.

08-02 Oprea, R., Wilson, B. and Zillante, A. War of Attrition: Evidence from a laboratory experiment on market exit.

08-01 Oprea, R., Porter, D., Hibbert, C., Hanson, R. and Tila, D. Can Manipulators Mislead Prediction Market Observers? 